pert such degradation and to promote justice, and this we shall now consider.

The first and essential requisite is, that expert witnesses should never be chosen by parties in contest, nor by their legal representatives, either in civil or in criminal trials. If possible, their opinions on questions at issue should not be known beforehand. I would suggest that the court be authorized by law to summon a suitable number of persons to act in the trial as experts, that they be questioned as to any possible bias in the case, and that counsel on both sides have the privilege to object on such grounds, as in case of jurors, but without privilege of peremptory challenge. The court, jurors and counsel on both sides ehould then have a right to question the expert. Such a plan would give him a judicial position, more satisfactory to himself and vastly more conducive to fairness and justice. The law should allow adequate fees, according to the value of the expert's time in his ordinary business, to be fixed in. the judgment of the court and included in the ordinary costs of trial.

I am clearly of opinion that legal counsel should not be allowed to suggest names of experts, nor to have anything to do in their choice beyond aiding in the discovery of any possible bias, and objecting for such cause. Neither should they be taken at hazard from a list of practitioners, as jurors are drawn from a polling list. A man competent to preside over a court may be trusted to select experts from the best of their class. In a city there is ample room for choice, but in a rural community it might be necessary to summon men from a distance.

The court should be authorized, in his discretion, to require one or more experts to listen to the testimony of ordinary witnesses, before giving their own opinions, for this course would obviously qualify them to understand the case and assist to a just and correct decision. This might be made optional with putting a series of hypothetical questions. In either event the same precautions should be used to guard against outside influence upon the experts as are taken with the jury, and for the same reason.

Since the foregoing was written, I observe that the Illinois State Medical Society has proposed a legislative act authorizing the judges of the Circuit and Superior Courts to appoint experts in medical and other sciences, for the purpose of giving opinions upon the evidence as presented in hypothetical form in both criminal and civil trials. The term of appointment is to be one year, and the judge may summon as many as three in one case. They are to be subject to cross-examination, but only upon points touched in their opinion. I think it safer that experts in an important trial be selected for the occasion and at the opening, so as to preclude their being tampered with by interested parties beforehand. Their opinions should be no more forestalled or anticipated than those of the court and jury.

This matter is one for each individual State to settle within its own jurisdiction; likewise for the national government within the purview of the federal courts. It would be well for a suitable example to be set by a Congressional act applying to trials in federal courts, and then we might hope that the several States would take like action so as to make the practice substantially uniform throughout the country. In my judgment it would be fitting and helpful for the American Medical Assocration to urge upon Con- gress some such measure as the one here briefly outlined, not only for the credit of our profession, but still more in the interest of justice.

\section{THE PREVENTION OF ASIATIC CHOLERA.}

Read in the Section on State Medicine, at the Forty-sixth Annual Meeting of the American Medical Association, at

BY ELMER LEE, A.M., M.D., Pr.B. chicago.

Asiatic cholera is one of the pestilential diseases that remains to menace the happiness and prosperity of the human race. In civilized countries epidemics are more and more rarely occurring, but cholera is still a dreaded and fatal disease in periodical epidemics of wide extent in civilized Europe. Today the disease prevails almost continuously in some portion of southern Europe and western Asia. The people in these countries are of two notable classes; those of the highest, but the majority of the lowest intelligence. Superstitions offer obstacles that so far have prevented the development of prophylactic measures against ravages of epidemic cholera. When a people such as are the occupants of southern Russia, threaten to murder the priest of the parish because he endeavors to introduce, for their use in times of cholera, so simple an agent as carbolic acid, it is not strange that preventive measures make slow headway. The Russian populace during the last epidemic of the year 1892, actually murdered a physician in cold blood, simply because of his profession. They believed that he, with other members of the profession, was indirectly the cause of their suffering and death. Mobs attacked the hospital barracks, dragged the patients into the open places, laid them on their backs in the hot sun, destroyed the hospital, threatened the lives of doctors and nurses, and in one instance a doctor was martyred. In another instance, in order to save his life, at the hands of these sadly superstitious people, it was necessary to place a physician in a coffin and carry him as if dead to the outskirts of the town. Even in more enlightened portions of civilized countries, grave difficulties present themselves to important measures relative to protection of human life in times of epidemics.

It is a long time since there has been an epidemic of the cholera in the United States, but it is perhaps an open question whether our own people in some instances would behave in the most enlightened manner. While it is agreed by the profession universally, that it is desirable to prevent epidemics, it seems everywhere to be scarcely possible to meet the laudable desire. Up to the present time, there have been and there are a number of propositions concerning the prevention of Asiatic cholera. The success of measures of prevention has not realized the expectations of the advocate who has proposed them. A most notable and widespread interest and enthusiasm was produced ten years ago by Ferran, of Valencia, Spain. The Doctor heralded to the world that by the use of his attenuated cholera germs, the disease was both quickly and surely curable, as well as preventable. His proposition received almost universal consideration, but to-day the proposition receives absolutely universal neglect. This is the sad history of the great hope that was extended throughout the civilized world, that at last a great man had discovered a method equal to the require- 
ment. Expectations ran high, the medical press, as well as the secular, was filled with seemingly strong and convincing facts that went far to establish the value of Dr. Ferran's proposition; but alas! as have so many propositions during our own brief generation, it waned and to-day is only remembered on account of the notoriety of the failure. Since his day, other equally intrepid investigators have had propositions to advance. Some of the propositions have been worthy of serious consideration, some of them have been ridiculous. In every instance the proposal to protect human life from the contagion of cholera has consisted of some foreign element to be introduced into the blood circulation. The principle underlying the introduction of foreign substances in to the circulation is that of antagonism. Some of these substances proposed to be introduced into the body have been animal substances, many of them mineral. The disposition of the moment, especially the disposition of European investigators, is along the line of animal viruses. These viruses are of two kinds: the newer kind is the sterilized toxins of the disease to be prevented; the older form consisted of attenuated bacilli. Both forms have sadly failed to prevent the disease feared.

The latest proposer of a virus of animal nature that would prevent Asiatic cholera is Professor Haffkine, of the Pasteur Laboratory, a Russian by birth and education. The Doctor is an intrepid experimenter, a faithful observer, and a profound bacteriologist. His great and untiring efforts seriously deserve a better fate than the results of latest experiments warrant. Encouraged by the hope of success, he has visited the home of Asiatic cholera in India, and a great many of the natives and members of the native army, were inoculated against cholera. The report of the results of his inoculations, show that 70 per cent. of the inoculated subsequently contracted cholera, whereas the percentage of the natives not inoculated who contracted cholera did not exceed 67 per cent. This is truly disheartening, but it was what was to be expected. Inoculation by animal viruses predisposes to Asiatic cholera, the opposite result to that which is desired. Inoculation against cholera has signally failed in the hands of each successive prophet. The virus subcutaneously introduced produced retrograde metamorphoses similar to the effect of the disease itself. The experiments in Calcutta by inoculation during the past three years have shown better results, but the conclusion is valueless for the reason that that community has not been visited by Asiatic cholera during the entire period of the experiment. If the attenuated germ and the sterilized product of the germs, or the sterilized product of the disease itself, after the conscientious trial of a generation fails, what hope remains along this particular line? There is nothing left to be tried, and it appears to me the case is hopeless in this direction. My own conviction honestly revealed, denjes any prospect of a realization of such hopes.

A still later earnest, intelligent, educated enthusiast in our own country comes forward with a new theory. Dr. Reginald Leach, a worthy and honored graduate of Dartmouth, proposes to prevent Asiatic cholera by a process of introducing arsenious acid into the circulation. His conclusion is the result of theoretical deduction. The proof of the value of the deductions has not yet been established, but the high standing of Dr. Leach entitles him to our most respectful consideration. He assumes, and in his assumption he has my concurrence, that to prevent a disease such as cholera, an agent is required which is in itself able to cure that disease. This is good reasoning; this is from my standpoint literally and actually true. Now, as to the selection of the remedy. The Doctor holds with me that the propositions of Pasteur, of Behring, Haffkine, Hammond, Ferran, and others of that class are faulty, because they use an animal toxin for their preventive measure. An animal toxin, the Doctor says, is necessarily uncertain in its dynamic force, owing to incidental and unavoidable causes. In addition to that, the morbid products of vicious character are liable to accompany the curative animal virus, owing to the impurity of the source from which it is to be taken. Consequently, Dr. Leach proposes to use a definite unvariable mineral of known strength, a mineral which can be weighed and the dose made exact. He reasons that there is some analogy between Asiatic cholera and malarial disorders, and that this mineral substance likewise is able to exert its peculiar protecting influence. Virchow has stated that there is some analogy between the effect of poisoning by arsenic and the disease, Asiatic cholera. Upon this basis of analogy, the theory is constructed in part, that that remedy which produces an analogic disease to cholera may be the remedy which will cure a prototype of that analogy. Another conclusion entertained is that while the morbid products of disease used as viruses for inoculation produce retrograde disturbances of the human body, on the contrary, arsenic is said by Bartholow, as the Doctor's authority, to produce tonic and reconstructive effects. Theoretically, this may or may not be true. Practically, the conclusion has not been determined. It is a serious question in my mind as to whether it ever can be proved to be true. The claims for prophylactic effect of arsenic are being investigated by certain colleagues in Lucknow and some other parts of India. Whatever may be the result, it is too much to hope that the prevention of Asiatic cholera can be secured by introducing any corrosive mineral substance into the circulation. Arsenic is an intense corrosive, and whatever its influence may be, its action is that of foreign corrosive substance. It has by right and by nature no natural place in the human organism. The reasoning from the point of theory by Dr. Leach is clear and deeply interesting and worthy of consideration. The whole subject has been plainly presented to me by the Doctor in the form of a personal letter, but it is too important a document to be retained by me as a private paper. It is my wish that it be included in the Transactions of this Section for publication in the regular way.

The prevention of cholera must necessarily be along two lines: 1 , the measures instituted and executed by the State: and 2, those means and measures to be carried out by the individual. The enforcement by the State of modern sanitation, modern hygiene, the principal feature of both the preceding, being a free supply of pure water for public and private use, constitute the wisest and the best provision as a public policy. The application of rules of strict and severe and unyielding quarantine measures is neither humane nor is it necessary.

Cholera is a preventable disease; but to prevent cholera requires coöperation between the directors 
and the directed. Education and the growth of intelligence alone will secure protective coöperation. What can we do in a place where its citizens, when commanded by the authorities to restrict their use exclusively to sterilized water, reply as they did along the banks of the Volga in Russia, that they will not do so because God Almighty did not intend them to drink boiled water, for if he had he would have boiled the water himself. The day when sufficient intelligence will inspire the motive for coöperation between the authorities and the masses is somewhat nearer but still a long way off.

Personal prophylaxis against cholera would avail much if the result of personal intelligent instruction. Victims of cholera are chiefly persons who are them- selves victims of vicious appetites or vicious habits. The excessive use of alcoholic beverages is without an equal as a chief factor in preparing the organism for an invasion of cholera. Excessive use of tobacco likewise weakens the system and invites cholera. Unscientific cooking and the too frequent use of animal foods are productive of a condition of body - which encourages cholera. The insufficient consumption of water, and especially pure water, is of all factors the most conducive to the acquirement of this disease. One preventive which would be a universal prophylactic against Asiatic cholera is the condition not as frequently found as it should be, which is known by the name of good health. Pettenkofer, of Munich, showed by his experiments upon himself, that with normal secretion of gastric juice, a tumblerful of cholera bacilli could be taken with mirth and impunity. Gastric juice is a corrosive substance secreted in the normal stomach, that attacks and cuts to pieces quickly and surely, every organic particle of matter that comes in contact with it. It is fully taught and fully believed that cholera is produced by organic germs which enter

- the stomach through the mouth in the contaminated water which is drank, or in the food which is consumed. The sentinel, in good health is vigilant and absolutely prevents every form of organic substance from passing the threshold into the human citadel. But if the guard who stands watch at the door is stupefied and rendered impotent by his own - vicious habits, he will permit the foe to enter, and the house to be invaded. Healthy tissue, together with a healthy mind, is the prophylaxis universal as to time and to place against every invasion of the system by Asiatic cholera.

It was my experience during a stay of six weeks in the Oboukoff Hospital in St. Petersburg, to see many cases of Asiatic cholera. There were 600 persons afflicted with cholera at the time of arrival. The cases of cholera were principally among the lower or lowest classes. These poor people live badly, eat poorly, and drink excessively; but instead of drinking pure water, they substitute a Russian liquor, and frequently while under its influence they drink water during the heat of the day from the filthy canals which divide the city into three parts. These canals are so foul that they resemble to some extent the Chicago River. Fish can scarcely be kept alive for even a few days in them. Very many of the men who came to the hospital sick from cholera, contracted the disease through their ignorant use of the contaminated water from the canals of the city. The preventive measure - that is universally employed, and which commended $i$ tself because of its good results, was the provision for a supply of boiled water for all the inhabitants. It is wise in observing to take note of a measure so simple, yet so generally recommended as the use of pure water during epidemics. This sensible measure is the only likely preventative of Asiatic cholera. During the prevalence of the epidemic in Hamburg, Altoona, a division of the city, was supplied from a different system of waterworks, from that of Hamburg, and while cholera raged in Hamburg to the extent of 21,000 cases in five weeks, there were no cases of cholera in Altoona, which was only separated from Hamburg by the difference in having a separate waterworks which supplied filtered water as against unfiltered water to the residents of the infected city. Pure water will save Hamburg from future epidemics of cholera.

\section{State Street.}

\section{IETTER FROM DR. LEACH.}

$$
\text { Paris, Texas, April 6, } 1895 .
$$

Dr. Elmer Lee, 100 State Street, Chicago, Ill.,

Iy Dear Doctor:-Your very welcome favor of the 3d inst. came to hand but shortly, and I hasten to reply that against your very large practical experience with the disease, I can not pit my remedy (as yet but partially tested) as a curative; nor do I so wish to do, for I hastily acquiesce in the verity of your special plea for absolute cleanliness in the treatment of Asiatic cholera, and I also have my apology to offer for some unmeant abstruseness which seems to have slipped into my letter of the $29 \mathrm{th}$ ult., for I did not then, nor ever, mean to commend arsenious acid, nor any other form of arsenic, as anything else than the remedy, par excellence, as a prophylactic against this disease; believing, always, that with absolute cleanliness, followed by the judicious exhibition of the proper drug, this disease may be reduced to the status of, at least, that now held by the more loathsome variola or possibly to something even better still, for I am at least sufficiently enthused with the belief in arsenization vs. cholera to make any reasonable sacrifice for the pleasure of personally testing the alleged efficacy of my drug to prevent; but to date I have been handicapped by a succession of misfortunes which have wellnigh buried me in that oblivion it seems the desire of the majority to relegate us, and the misfortune of all such who are adventurous in medicine to that point where those who dare but follow pronounce each temerous.

I assure you, therefore, that I appreciate to the utmost, the honor of the attention you have thus far bestowed upon my hypothesis, and trust it may be my good fortune ere long to forward you some more convincing news from the East, of the efficiency of my chosen drug as a prophylactic against this fell destroyer of our fellow beings; meanwhile, I trust you will excuse me when I acknowledge a timidity in asserting views diametrically opposed to the generally accepted theories of the day as presented by Pasteur, Haffkine. Hammond, Behring and others, but at the same time accord me the privilege of the paradoxical position of ap. parent temerity in asserting my views on arsenization as the only true prophylaxis against cholera (as you have seen it) I believe logical reasoning can arrive at; and accredit me with the desire only to better our standing as preventers of disease, however attained.

As you say, my theory is clever and engaging, please do me the further honor of reading something more of the same which will more fully acquaint. you with the wherefore I am so confident of its ultimate good to mankind; and then, if you please, reward me with your further correspondence upon this very intensely interesting subject.

Granting that you will continue to read to the end, I take great pleasure in stating that I find, in reading up on $m y$ opponents (inoculation of animal virus) that Toussaint and Chaveau first recognized the fact that not only the attenuated disease germs, but also the sterilized products of bacterial life, could communicate immunity, but Behring says the acquired immunity is of a relatively short duration and the dismal results of Haffkine at Lucknow show that he there lost over 70 per cent. of the inoculated, while there was a mortality of less that 67 per cent. among the uninoculated, showing a morbid condition we would wish to aroid in alleging prophylactic virtues of anything; therefore please note that while I shall attempt to show the fallacy and even dangers of other hypotheses on the prevention of Asiatic 
cholera, I do not do so till I feel confident I have something logically correct as a substitute; and, therefore, not as an iconoclast but as a simple investigator do I propound $\mathrm{mg}$ own theory to you as to all others, hoping only now to sufficiently interest those who may have the ear of public opinion, that tests may be made where such is possible, and the sooner the better, as the more certain of something tangible and reliable.

We know that Virchow first promulgated that "many cases of acute arsenical poisoning are not distinguishable by their symptomatology or morbid anatomy from cases of epidemic cholera;" that Farrington proclaims that arsenic "excites intestinal disease which is almost identical with cholera, even the organic growths of cholera are found in the discharges from the arsenic proving ;" also that since 1873 sulphuric acid (as I wrote in my last) has held a high place among the alleged preventive measures against this form of cholera. Therefore in handling this much mooted question I ask your forbearance and a reservation of verdict until I have presented more testimony than I felt it my privilege in my letter of the 29 th ult.; and in doing this you will please note I shall attempt to faithfully present all sides of the question; at the same time claiming most for arsenic as the preventive of the disease, as well as the superiority of drugs over all kinds of animal viruses as preventatives, because I am with you in my conviction that the whole prineiple now underlying allopathic practice is incorrect, and I say so though I am a graduate of Dartmouth College also that I do not now follow the precepts of my alma mater.

Though this may seem already long I have but just begun, yet shall try and not burden you with a novel method of cure, but only present a prophylactic measure as different from a curative as is hygiene from medicine, ye $t$ as allied in significance and utility. I follow the same general trend of thought as that of all our illustrious predecessors in preventive medicine (for there is but one royal road to success) but am constrained to choose a drug instead of their inoculation with the animal viruses or antitoxins, for I choose more measurable substance, which, in exhibition as a prophylactic against cholera, is within our power to control by regulating the dosage and within our ken in most probable effect; at the same time eliminating possible idiosyncrasies in persons of unusual susceptibility; but even in them prepared to meet any untoward symptoms with a known antidote such as we have not for the animal viruses.

I choose a drug because of the fact that extraneous noxious agencies (such as natural diseases and animal viruses) possess subordinate and of ten extremeiy conditional powers, while drugs possess absolute and unconditional powers far superior to the former in their ability to produce morbid discordancy or the antitype of the prominent, uncommon and eharacteristic signs and symptoms equal in number to the disease to be prevented which must be the power of any protective to protect. I choose arsenic, therefore, because, as Hippocrates says: "Experience has demonstrated that only that remedy will prevent a disease that possesses the power of curing the disease feared, and which can produce reaction in the system which is directly antagonistic to the disease and its influences." Though I do not claim that the inoculation process with the sterilized viruses will not cure the disease feared, I do assert that in a system that might otherwise demonstrate cholera, that inoculation with animal viruses can not produce reaction directly antagonistic to the disease and its influences, but on the contrary, with cholera, I claim that the inoculation process (contrary to the same process in smallpox) weakens and makes more susceptible to the influences of the natural disease each one inoculated, and by virtue of its similar powers of destructive metamorphosis to the action of the disease, epidemic cholera.

I choose arsenic, also, because long before the profession was dominated by the manufacturing chemist, arsenic was known to be one of the most potentremedial agents in combating this disease, and is even now quoted by Bartholow as " one of the numerous remedies proposed for the cure of this disease," and by others as "indicated in all stages of the disease; "and as it is tonic in effect and a reconstructive, both of which are diametrically opposed to the destructive metamorphosis at work in the cholera patient or him likely to become such. I choose my drug also because, as $11 \mathrm{~m}$. Henry Porter says: "The arsenites (that is the molecular elements which constitute the arsenites) are intensely irritating and poisonous to all forms of protoplasmic life, both animal and vegetable. At the same time they enhance the accumulation of tissue throughout the whole animal economy, and when this is done diseased processes all through the system are in part or completely removed and more or less of a new normal or healthy activity is brought to all parts of the body."

I choose arsenic, also, because authority and experience teach us that the human economy is more susceptible to drug action and the vital force more responsive to its magic touch than to any miasm or perversion of nature's laws ; therefore, while I acknowledge that sterilized products of bacterial life might communicate a like immunity, accord me the privilege of stating that many may be the imperfect results of chemic action in this process rewarding us with such prognoses as awaited Haffkine at Lucknow and Lady Montague in England where, in either instance, the results were such as could not be predicted by the most gruesome pessimist of that time or of this; affecting as they did such monstrous mortalities that thanks to Jenner many were saved.

As Behring says of his antitoxin: "The acquired immunity is of a relatively short duration," so I assert of Haffkine's inoculation, and likewise acknowledge a probable short immunity from arsenization, for I accept authority for the statement that " the effects of arsenic continue in the system from a few hours to four weeks, or longer, according to the size and frequency of the dose," yet while the effects of inoculation are not known positively they are of arsenic, and so far as jet demonstrated the results show for the former more of destruction than protection, while for arsenic more for protection and none for destruction. In this, I take no account of the 200 cases reported by Simpson from Calcutta, where there had been no danger of infection for over three years previous to his inoculations. Such misleading reports can have no effect, pro or con, upon the actual prevention of this disease, and their publication as made in American newspapers and medical journals is simply wrong, to say the least; for a prophylactic to command the courtesy of the name must protect a majority of those alleged to be protected, and only where disease and danger to infection is active and imminent and not in situations where it may have existed in the past but has not since reappeared.

In such times as these, therefore, when we are seeking after the true preventive for each preventible disease, with a loyal but misguided enthusiast in the Orient sending to our Western civilization only the immediate results of a most pernicious though seemingly very plausible theory, we can and do realize and recognize the sacrifices of the investigator into the occultness of disease, more especially this of epidemic cholera, yet only to the successful discoverer of that protective which will protect all, be that what it may, can we honestly render our respectful acknowledgments. To return to the why and the wherefore of the proposed substitution of arsenic for all others as a preventive of cholera we recall the facts relative to cases of supposed cholera at Cincinnati in September, 1892, and of similar cases at Little Rock in the following month, wherein the auxiliary diagnosticians of the microseope and chemic analysis were required; and in the first instance showed similar though not identical germ life to Asiatic cholera in the milk drunk by those victims, while in the second instance chemic analysis demonstrated arsenical poisoning at Helena while the microscope showed the Little Rock cases to be those of so-called winter cholera. In all extmplifying bacteriology the acme of diagnosticians is germ diseases (after the germs are admitted) and Asiatic cholera and arsenic poisoning are, as Virchow so aptly states, indistinguishable (oftentimes) by their symptomatology and morbid anatomy from each other (except, as stated,) by the auxiliaries here noted.

If these be facts, and I believe all know them as such, then truly a drug is eminently superior to any animal virus or if there can possibly be a better process than the one of Haffkine or Ferran (with the excreta of the cholera patient, sterilized or not) why not a drug which we have known in effect for the past century, instead of risking a repetition of our certainty to infection with the disease feared, as well as some possible worse disease. Experience has shown recently in India that there is more danger to the inoculated than to those where no precaution against infection was made, and remembering the results of inoculation from 1721 to 1783 and that the process of sterilizing can be unsuccessful at times (the cause of Haffkine's late failures) then why place ourselves and patients in the unfortunate position of Lady Montague and her enthusiastic collaborators, when they produced (of course unintentionally), a condition which may best be described by that trite old simile of the early English and known as: "the groans of the Britons," in which they are made to say: "The barbarians on the one hand drive us into the sea; the sea, on the other, throws us back upon the 
barbarians; and we have only the hard choice left us of perishing by the sword or by the waves."

If this appears far-fetched, my dear Doctor, look at the results in Haffkine's Lucknow inoculations in which it was supposed the sterilization of the virus there used was faulty, and if such was the case there, it is but fair to presume that in any of the "hair splitting" processes of eliminating individual or family characteristics or diseases from the excreta of the cholera patients, we may never have a truly sterilized immunizing virus containing only the comma germs and their toxic results or combinations. For example, let us look upon an imaginary threatened epidemic of the disease and in the following personal manner, wherein you will imagine yourself in imminent danger to infection because your nearest neighbor has the disease. You each use the same well for obtaining your supply of drinking water and your families exchange the ordinary courtesies of such relationship; but you know him or at least suspect him the domicile of syphilis, scrofula or tuberculosis. This is the only case thus far, and inoculation as now promulgated, the only preventive measure known to the profession.

If Scylla and Charybdis presented such fearful obstacles to the usually undaunted ancients, which of these seems the more favorable to you? the Scylla of the hardened syphilitic or the possible swirling, twisting and contorting Charybdis of Asiatic cholera? for in this, maybe a little overdrawn simile, you see inoculation against cholera as it might exist and the consequent predicament of our people. This is in fact the only view to take of inoculation as it exists, as shown by the grievous experience of our colleague in India; yet, despite all this, we find the leaders of preventive medicine of to-day forgetful of the near past, and the wave of a more certain and accessible prophylactic which is offered in arsenization, of which I had the pleasure in $\mathrm{my}$ letter of the 29th ult, of reporting so much of what is good and none of a like character to the inoculations of my unfortunate colleague.

If, then, Pasteur prevents hydrophobia with a virus which presents symptoms in the inoculated almost identical with the rabies canina, and Jenner's vaccine may, and sometimes does, exhibit such a similarity to true smallpox as to question the purity of the virus, and it be a fact, as Virchow says, that arsenic produces a condition which may preclude a positive differential diagnosis with cholera to produce this effect, then the arsenious acid must actually occupy the exact space or place in the animal economy or affect the intestinal pabulum in a like manner (as I think I wrote you before) as would true Asiatic cholera, (and it matters not which is the solution) to there demonstrate its certain effect. Therefore, by the willful arsenization of men up to a condition of slight physiologic effect of the remedy, such, for instance, as is produced by the animal virus, this process must and will effectually prevent that one so treated simultaneously demonstrating in himself the disease feared, and just as surely, and upon exactly the same hypothesis that we presume and logically conclude that the vaccinated can not suffer simultaneously the full virulence of variola; yet in this we need not be more void of common sense in our expectations of arsenization than of vaceination (already sufficiently tested to satisfy a big majority) as facts simply verify, in the latter, that personal idiosyncrasy in the well is quite a factor in the equation, and that such affects its prognosed results as in the administration of different remedials to the sick. Therefore not every one arsenicized, more than every one vaccinated, will be protected against disease more than is to be expected of otherwise positively indicated remedies prescribed, as they all are, according to our fallible diagnostic powers and prognosing hopes.

These, then, are some of $\mathrm{my}$ reasons for promulgating what Gibier so aptly pronounces my "theoretically perfect" hypothesis, and the reason for my choice of a drug in preference to any animal virus. Because $T$ believe that through a drug alone, exhibited into the system and that system made responsive to its physiologic effect, and so kept while danger lasts, may we be protected, and finally emancipated from the ravages of epidemic or Asiatic cholera. By this method alone may we hope for relief from the pernicious theory of inoculation advocated by the gentlemen referred to; and by drug effect alone may we hope to keep off indefinitely the possible dangers far worse than the probable epidemic disease; and by drug effect alone practice truly aseptic prophylaxis against this disease.

If, then, the sterilized products may become dangerous through some unknown cause, why should we advocate further a process which, at best, can but effect an equal immunity with arsenious acid which latter is long known as

to effect, where positive antidotes are the public property of all inquiring minds and named in all medical works pertaining to the subject of arsenic and its compounds. Why, then inoculate at all, while the alleged acquired immunity is of so relatively short duration, and while a more certain and safer effect may be had by arsenicizing, which demonstrates truly one more long known fact that two diseases, though different in kind (drug and miasm) but very similar in their manifestations of signs and symptoms will always extin. guish each other, whenever they meet in the system, for the superadded morbific potency (drug) takes possession chiefly of the same parts in the organism heretofore affected (or hereafter to be affected) by the weaker morbific agency (disease). This is thereby deprived of its power of action and is consequently extinguished or prevented.

In arsenization we therefore have a safe, sure and accessible prophylactic ; for such a process must demonstrate true asepsis and the power to produce a like condition to that intended to prevent, as well as a condition directly antagonistic to the disease and its influences. All of which Bartholow unconsciously affirms when he says: "Arsenic is, with but a few exceptions, destructive to animal and vegetable germ life and is a reconstructive and a tonic to the system, yet in lethal doses produces symptoms similar to epidemic cholera." To all this I add that I note with pleasure that you agree with me that Haffkine's inoculation theory, like Ferran's, must fail under test, but what must also be the verdict of all others in the case of inoculation as practiced, filled as it must be necessarily with provisional possibilities of other and worse diseases than Asiatic cholera. What of this inoculation process in comparison with arsenization which must not only protect the inoculated or arsenicized, but also protect all coming into his presence, as well as those who, by virtue of their environment, might ingest particles of his ejects? In the arcenicized is one less germinating center and one less germinating medium for the rapid incubation and dissemination of the comma bacillus and its toxins, thus sterilized beyond any possibility of a doubt and the result unaccompanied by any wail of possible failure in the process, such as came from India after the demonstration of such a fearful mortality.

Therefore, if we are to have protection against cholera through a process yclept vaccination or inoculation (as predicted by von Pettenkofer), and none can be more efficacious or expeditious, it can be done in but the one way, and that is by arsenization of each and every suspect or patient (in the first stage), or person subjected to possible infection, as well as to all others seeking protection, and this should be repeated after four weeks at the longest if danger to infection remain. So by arsenicizing with ivory points charged with doses of the one-thirtieth of a grain of pure arsenious acid (such as are made at the Lancaster County Vaccine Farms), each one thus brought under the sufficient effect of the remedy may experience a successions of symptoms resembling an attack of cholera in miniature, yet with few, if any, of the distressing symptoms usually incident to true cholera. At most, there will be but slight diarrhea, with burning at the epigastrium and under the eyelids, and a ringing and roaring in the ears, as is common after dysentery, diarrhea, or profuse fluxes of any sort; but in inoculation with the offal of the cholera patient most of these symptoms are more pronounced in that each must, perforce, sufer a mild and sometimes even a virulent attack of the actual disease. Under slight physiologic effect of arsenic, one has but taken a dose and acquired its effects, such as has been common practice (in prescriptions during the past century) for chronic malarial cachexia and skin diseases of a germ origin, and is still an almost diurnal prescription for such maladies with the "regular" doctors.

Such constitutional effect of the drug may be produced when there is no especially imminent danger to infection, by the ingestion of from 2 to 5 drop doses of Fowler's solu. tion, repeated every two hours till the desired effect is acquired; or the same may be effected by hypodermic administrations in like dosage and repeated until like effect is acquired; but all this. like successtul vaccinations, should be done only under the prescription and immediate supervision of the physician, who holds ever ready, in dialyzed iron, an antidote to any possible idiosyncrasy in the applicant; and such none may possess against the inoculations with any degree of certainty of action; for once inoculated or ingested, the cholera germ or miasm takes its own course, and often the life of him experimented upon.

From some comes the common wail of fear for those thus arsenicized, but to all I respectfully request a comparison of the mortality at Berlin alone, before and after the intro- 
duction of vaccination, yet this process of protection counts some victims. Compare the mortality from cholera and from Haffkine's inoculation and that from arsenic poisoning (in the hands of the physician when purposely prescribed); compare also the possible dangers incident to inoculation (even to those who survive the process) to the process of arsenization as I propose it, and of all these choose the lesser evil, if evil it be to try and avoid disease; compare the actual increased mortality from inoculation against that where it was not practiced at all and therein alone see the fallacy of a theory in relation to the practice of which we speak; and then remember that inoculation calls for a first case, with all its possible accompanying dyserasiæ, its entire lack of effective antidotes and its frequent mortality. While arsenization calls for the ingestion or other manner of acquiring a slight physiologic effect of arsenious acid (a drug in daily use the whole world over), and that in this process the re need be no first case from which to obtain the virus, for this drug is known to all medical men, who know its effects and its antidotes, while few know cholera and its effects, and fewer know its antidotes.

Let me, therefore, my dear Doctor, review your favor of the $3 \mathrm{~d}$ inst., and remind you first that where you find the consensus of opinion to have been against good results from the use of drugs during the epidemic in Europe in 1892, that I believe you have only been able to hear fro $m$ one side of the profession, and that side used much too large doses, thereby augmenting the diseased condition by adding drug disease, whereas by the judicious administration of much reduced doses jou would have probably come out of that epidemic much more in favor of continuing the good oldfashioned drug medication. I am also truly pleased to find one more who shall acknowledge, with you, that "adding to an already overburdened and disordered blood new and additional foreign and disturbing substances is the principle underlying allopathy, but it is not the correct principle involved in curing disease." I would respectfully cite you to one Professor Brunton (medical author, editor and examiner), who acknowledges a like empiricism in the allopathic practice, which is claimed to be the only scientific process of medicating our fellows; and as above stated I am pleased to add that I am one with you in encouraging absolute cleanliness in the cholera patient. But do not forget that drugs will do much more than the majority of practitioners. I now respectfully and forcefully present my allegations in detail and shall appreciate much your further consideration of this most interesting subject, especially as I can see that through your disinterested interest you further promulgate this hypothesis, which is thus far "incontrovertible except by test," and which has thus far stood firmly against all adversities.

I conjure you, my dear Doctor, to write of this subject while you have the ear of the entire profession, as I am but yet little known; also do me the honor of an early answer and your candid opinion as to the theory as thus far propounded, and believe me, Yours very truly,

REg. Leach.

\section{THE PROPRIETARY SYSTEM AND ITS} REMEDY.

Read in the Section on State Medicine at the Forty-sixth Annual Meeting of the American Medical Association, held at Baltimore, Md., May $7-10,1895$.

BY F. E. STEWART, M.D., Ph. G. DETROIT, MICH.

THE PROPRIETARY SYSTEM.

For the purpose of promoting progress in science and the useful arts the Constitution of the United States gives Congress the power to grant to authors and inventors for limited times the exclusive use of their respective writings and discoveries. Hence our copyright and patent laws.

A patent is a contract between the government, representing the public at large, and the inventor. The consideration moving from the government is the granting of the exclusive use of the invention for a limited time. The consideration moving from the inventor is the invention of a new and useful article, and the giving to the public full knowledge of it by a proper application for a patent, so that, when the pat- ent expires, the public may manufacture the invention. The wisdom of such a law seems apparent, for by it inventors are stimulated to produce new and useful articles, capital is protected during the working and marketing of the invention until the investment becomes remunerative, knowledge is increased, and the archives of the Patent Office become a great public library and industrial exhibit, free to the use of the public, and commerce is promoted by valuable additions to the list of marketable commodies.

A trade-mark is a "commercial signature" employed to distinguish one brand of an article from another brand of the same article on the market. Its use does not confer on the user any exclusive right to the manufacture and sale of the article upon which the trade-mark is branded. In law, the trade-mark has two uses. One is to designate ownership, illustrated by the common method of branding horses and cattle so often seen on the ranches of the great West. Another use is to designate the source of emanation or of manufacture. For this reason the lumberman brands his logs with a trade-mark, or the manufacturer of pottery stamps his wares with peculiar marks or signs. In the same way the manufacturers of silk, linen and other fabrics use the words "Lyon," "York Mills," etc., as trade-marks, to distinguish their various brands of goods from other brands of the same articles on the market. But these uses of the trade-mark do not vest in the users the right to the exclusive handling, or manufacture and sale of the articles upon which the trade-marks are branded. The man who brands his cattle with a trade-mark does not acquire thereby a monopoly in cattle; neither does the use of trade-marks by potteries, silk manufacturers, makers of linen and other fabrics, vest in the users the right to exclude others from making and selling the same commodities.

Another use of the trade-mark has sprung into being as a development of modern times-a use not contemplated by law. The method to which I refer is the registering at the Patent Office of the only name by which an article is known, as a trade-mark on said article. This use of the trade-mark has created what is known as the proprietary system. This system should not be confounded with the patent system, as it is, in principle and practice, directly opposed to it, as a further consideration of the subject will show.

The monopoly created by registering the only name by which an article is known as a trade-mark upon such article is unconstitutional, illegal and unscientific. These charges I now proceed to prove.

The monopoly is unconstitutional because unlimited in nature. Congress has no power to grant perpetual monopolies. I grant that the right to use a trade-mark can not be limited by law. But, as I said before, when one puts his commercial signature on his goods he acquires thereby no exclusive right to the manufacture and sale of articles so marked. No monopoly whatever can result from the proper use of a trade-mark. Only its misuse or abuse creates the so-called proprietary system.

The monopoly is illegal, for there is no law in the statute books of the United States designed to protect the new use of the trade-mark. On the contrary, the courts have decided that when a new article of commerce is born it must be provided with a name; and that any one has a perfect right to manufacture and sell the article under the name by which it is 